\title{
Euphrasia-Augentropfen bei Neugeborenen: Ein Pilotprojekt
}

\author{
Lilian Stoffel', Doris Zimmermann², Romi Hunkeler1, Claudia Zimmermann1, Mac Ramos³, Martina Fathi-Torriani1, \\ Gaby Kropf2, Mathias Nelle1 \\ 1 Universitäre Medizinische Kinderklinik, Inselspital CH-Bern; 2 Universitäre Frauenklinik, Inselspital, CH-Bern; 3 Weleda AG, CH-Arlesheim
}

n der universitären Frauenklinik Bern kommen jährlich zwischen 1200-1300 Kinder zur Welt [1], davon sind ungefähr $7-10 \%$ Frühgeburten [2]. In der Abteilung Neonatologie werden sowohl gesunde Frühgeborene wie auch kranke Früh- und Termingeborene betreut, die entweder in der Frauenklinik oder extern (Universitätsklinik, Bezirkspital, Geburtshaus usw.) geboren wurden.

Bei Frühgeborenen wie auch Termingeborenen $^{4}$ kann in den ersten Lebenstagen bzw. Wochen nach der Geburt ein verminderter Abfluss von Augensekret via Tränengang auftreten. Dadurch entwickelt sich häufig ein Sekretstau mit den Symptomen gerötete, tränende oder fliessende Augen, mit Bildung von weissem oder gelbem Sekret. Standardgemäss werden die Augen mit NaCl 0,9\% ausgewaschen. Bei einer Verstärkung der obengenannten Symptome kann dies zu einer Infektion der Augen führen.

Eine Augeninfektion in der Neonatalphase wird in der universitären medizinischen Kinderklinik Bern mit Neosporin-Augentropfen ${ }^{\circledR}$ behandelt [3]. Neosporin-Augentropfen ${ }^{\circledR}$ enthalten eine Kombination von drei bakterizid wirkenden Antibiotika, nämlich Polymyxin B, Neomycin und Gramicidin [4]. Unter diesen drei in Neosporin-Augentropfen ${ }^{\circledR}$ enthaltenen Antibiotika ist besonders Neomycin aufgrund seines bekannten erhöhten otound nephrotoxischen Potenzials sowie seiner erhöhten Resorption bei Kindern unter zwei Jahren zu nennen. Ein

\footnotetext{
4 Im Weiteren werden sowohl Frühgeborene wie
} auch Termingeborene als Neugeborene bezeichnet.
Hintergrund: Bei früh- und termingeborenen Kindern tritt häufig in den ersten Lebenstagen bzw. -wochen nach der Geburt ein verminderter Abfluss von Augensekret via Tränengang auf. Dadurch kann sich ein Sekretstau entwickeln, der zu einer Infektion der Augen führen kann. Eine Augeninfektion in der Neonatalphase wurde bis anhin mit Neosporin-Augentropfen ${ }^{\circledR}$ behandelt. Bei Kindern unter zwei Jahren besteht die Möglichkeit einer erhöhten Resorption und damit eines erhöhten oto- und nephrotoxischen Potentials von Neomycinsulfat. Studienziel: Untersuchung des Einflusses der lokalen Applikation von Euphrasia-Augentropfen auf den Antibiotikaverbrauch bei Neugeborenen. Material und Methode: 24 früh- und termingeborene Kinder erhielten eine lokale Applikation von $\mathrm{NaCl}$ 0,9\% plus Euphrasia-Augentropfen. 20 früh- und termingeborene Kinder der Kontrollgruppe wurden konventionell mit $\mathrm{NaCl} 0,9 \%$ behandelt. Ergebnisse: Bei einer Behandlung mit $\mathrm{NaCl} 0,9 \%$, aber auch mit $\mathrm{NaCl} 0,9 \%$ plus EuphrasiaAugentropfen, konnte in den meisten Fällen auf eine Antibiotika-Therapie verzichtet werden, vor allem bei positiven bakteriologischen Abstrichen mit Staphylococcus aureus. Eine Limitation dieses Pilotprojektes zeigt sich in den Strukturunterschieden sowohl der Neugeborenen wie auch der Augenabstrichresultate. In der Versuchsgruppe wiesen die Neugeborenen ein tieferes Geburtsgewicht und Gestationsalter auf und waren tendenziell klinisch instabiler. Schlussfolgerung: Bei einem positiven Augenabstrich ist eine Therapie mit einem Antibiotikum nicht in jedem Fall notwendig. Ein Kind sollte nicht immer primär mit antibiotikahaltigen Augentropfen behandelt werden.

Schlüsselwörter: Prospektive Studie, Neugeborene, Augeninfektion, Euphrasia-Augentropfen

\section{Euphrasia Eye Drops in Neonates: A Pilot Project}

Background: Both pre-term and full-term neonates frequently exhibit reduced flow of eye secretion through the tear duct shortly after birth. This can cause congestion of the secretion, possibly leading to an infection of the eye. Neonatal eye infection has till now been treated with Neosporin Eye Drops ${ }^{\circledR}$. This may cause increased absorption in children, which would increase neomycin sulfate's potential ototoxicity and nephrotoxicity. Objective: To investigate the effect of local application of Euphrasia Eye Drops on antibiotic consumption by neonates. Material and Methods: 24 preterm and full-term children were given a local application of $0.9 \% \mathrm{NaCl}$ plus Euphrasia Eye Drops. 20 full-term and pre-term children in the control group were given the conventional treatment of $0.9 \% \mathrm{NaCl}$. Results: By treatment with $\mathrm{NaCl} 0,9 \%$ but also with $\mathrm{NaCl}$ 0,9\% plus Euphrasia Eye Drops an antibiotic therapy can be avoided, particularly in presence of positive bacteriological smears with Staphylococcus aureus. This pilot project was limited by the structure difference of both the neonates and the eye smear results. The neonates in the test group had a lower birth weight and a lower gestational age. They also tended to be clinically less stable. Conclusion: The results indicate that antibiotic treatment is not always to be regarded as necessary, even if the eye smear is positive. A child should not routinely be given primary treatment with antibiotic eye drops.

Key words: Prospective study, neonates, eye infection, Euphrasia Eye Drops 
Cochrane-Review zeigte einen kaum erkennbaren, grenzwertigen Gewinn von lokaler Antibiotikaanwendung im Vergleich zu Placebo [5,6]. Eine aktuelle Studie belegt, dass eine sofortige lokale Antibiotikatherapie das Wiederauftreten der Konjunktivitis sogar begünstigt [7].

Zubereitungen aus Euphrasia officinalis L. (Augentrost) werden seit dem 16. Jahrhundert in der Augenheilkunde eingesetzt. Die Pflanze enthält unter anderem Iridoidglykoside wie Aucubin und Euphrosid, Gerbstoffe und Flavonoide. Bisher wurde keine toxische Wirkung des Augentrostes bzw. aus ihm gewonnener Auszüge beschrieben. Euphrasia-Augentropfen enthalten keine synthetischen Konservierungsstoffe. Sie wirken antimikrobiell, entzündungshemmend, adstringierend und leicht schmerzlindernd. Entsprechende Präparate sind in der Schweiz als Arzneimittel in der Liste D (freiverkäuflich in Apotheken und Drogerien) registriert. Euphrasia-Augentropfen werden bei geröteten, tränenden und/oder fliessenden (weissem/gelbem Sekret) Augen eingesetzt. Bei bestimmungsmässiger Anwendung sind keine unerwünschten Nebenwirkungen bekannt [8].

Studien mit Euphrasia-Augentropfen wurden bei Erwachsenen und Jugendlichen durchgeführt. Sie zeigten positive Ergebnisse; unerwünschte Arzneimittelwirkungen traten dabei nicht auf [9,10]. Die Literaturrecherche mit den Schlagwörtern „conjunctivitis“ AND „neonate or newborn“ in der Datenbank MedLine ergab keine Studienergebnisse mit Euphrasia-Augentropfen bei Neugeborenen.

Mit dem vorliegenden Pilotprojekt sollte geprüft werden, ob sich bei Neugeborenen der Einsatz von Antibiotika durch lokale okulare Applikation von NaCl 0,9\% plus EuphrasiaAugentropfen D3 (WELEDA AG, Arlesheim) beziehungsweise $\mathrm{NaCl} 0,9 \%$ alleine reduzieren lässt.

5 Das Untersuchungsprotokoll wurde durch Face Validity durch den medizinischen Abteilungsleiter und die Pflegeexpertin der Abteilung Neonatologie der Universitätskinderklinik, Inselspital Bern, geprüft.

\section{Material und Methode}

\section{Design}

Das Pilotprojekt wurde als eine prospektive, randomisierte, kontrollierte offene Untersuchung ohne Fallzahlplanung angelegt. Die Randomisation erfolgte mittels Losziehung. Die Lose befanden sich in einer geschlossenen Schachtel.

\section{Ethische Überlegung}

Die interne ethische Kommission der Abteilung Neonatologie, bestehend aus dem Abteilungsleiter, der Leitenden Pflegefachfrau, der Pflegeexpertin, je einem Oberarzt und einer Stationsleitung, erteilte die Bewilligung zur Durchführung dieses Pilotprojektes. Die Eltern der in der Untersuchung eingeschlossenen Neugeborenen wurden mündlich und schriftlich informiert und gaben mündlich ihr Einverständnis. Die ethische Überlegung war, dass $\mathrm{NaCl} 0,9 \%$ bei symptomatischen Augen als medizinischer und pflegerischer Standard angewendet wird und das Anbieten der EuphrasiaAugentropfen ein Mehrgewinn dank der physiologischen Wirkung sein könnte.

\section{Datenerhebung}

Die Untersuchung wurde im Zeitraum 1. Januar 2005 bis 31. Dezember 2005 durchgeführt. Die Daten wurden mit einem eigens entwickelten Untersuchungsprotokoll 5 und nach selbstentwickeltem Ablaufschema erhoben (Abb. 1). Das Untersuchungsprotokoll wurde von den involvierten Pflegefachpersonen ausgefüllt (Visiten).

\section{Kollektiv}

Es wurde ein randomisiertes Pilotprojekt mit einer Kontrollgruppe durchgeführt, wobei eine Stichprobe von 48 Neugeborenen angestrebt wurde.

\section{Einschlusskriterien}

Neugeborene

- im Inselspital Bern geboren,

- unabhängig ihres Alters und des Gewichtes,

- unabhängig davon, ob sie unter einer systemischen Antibiotika-Therapie stehen,

nit tränenden Augen, mit geröteten Augen,

- mit fliessenden Augen mit weissem oder gelbem Sekret.

\section{Ausschlusskriterien}

- Neugeborene mit einer Sepsis

\section{Protokoll}

Vor Therapiebeginn und vor einem Wechsel auf Neosporin-Augentropfen ${ }^{\circledR}$ wurde bei jedem Neugeborenen ein Augenabstrich durchgeführt. Die Abstriche wurden auf Chlamydien und Bakterienwachstum untersucht.

Bei der Kontrollgruppe wurden die Augen alle sechs Stunden mit $\mathrm{NaCl}$ $0,9 \%$ ausgewaschen.

Bei der Versuchsgruppe wurden die Augen alle sechs Stunden mit $\mathrm{NaCl}$ 0,9\% ausgewaschen und beidseits je ein Tropfen Euphrasia Augentropfen in den Bindehautsack verabreicht.

Bei beiden Gruppen wurden die Augen mit einem sterilen Mesoft-Tupfer und $\mathrm{NaCl}$ 0,9\% von aussen nach innen ausgewaschen. Die Durchführung der Augenpflege erfolgte durch instruiertes, ausgebildetes Pflegefachpersonal. Ein Wechsel auf NeosporinAugentropfen ${ }^{\circledR}$ erfolgte nur nach $\mathrm{Ab}$ sprache mit dem behandelnden Neonatologen (Abb. 2).

\section{Beurteilung}

Konnte die Behandlung nach 48 Stunden als „erfolgreich“ beurteilt werden, wurde sie bis zum Abklingen der Symptome weitergeführt. Wurde die Behandlung als „nicht erfolgreich“ beurteilt, fand eine Umstellung auf Neosporin-Augentropfen ${ }^{\circledR}$ statt. Kriterien für die Umstellung waren eine Verschlechterung der Symptomatik und/oder ein positives Augenabstrichresultat. Vor der Umstellung wurde ein weiterer Augenabstrich veranlasst.

\section{Statistik}

Aufgrund der verschiedenartigen Stichprobenzusammensetzung (sowohl gesunde Früh- oder Termingeborene wie auch kranke Früh- oder Termingeborene) wurde eine deskriptive Datenanalyse (Median, Mittelwert, Häufigkeit) gewählt und mit dem Statistikprogramm SPSS 11.0 ausgewertet. 
PD Dr. med. M. Nelle

Abteilungsleiter Neonatologie

Lilian Stoffel
Pflegeexpertin
MINSELSPITAL

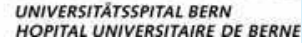

\section{EVALUATION EUPHRASIA AUGENTROPFEN PLUS NACL 0,9\% VERSUS} NUR NACL $0,9 \%$

\begin{tabular}{|c|c|c|c|}
\hline \multirow[t]{2}{*}{ Euphrasia-Augentropfen und $\mathrm{NaCl} 0.9 \%$} & $\begin{array}{l}\text { Name Kind/ } \\
\text { Geschlecht }\end{array}$ & weiblich & männlich \\
\hline & Aktuelle SSW/L & & ........ \\
\hline Nur $\mathrm{NaCl} 0.9 \%$ & $\begin{array}{l}\text { Diagnose } \\
\text { Antibiotika } \\
\text { Station }\end{array}$ & nein & ja \\
\hline
\end{tabular}

\begin{tabular}{|c|c|c|}
\hline & Auge Rechts & Auge Links \\
\hline \multirow{2}{*}{$\begin{array}{l}\text { Beurteilung } \\
\text { Ausgangslg. } \\
\text { (0 St.) }\end{array}$} & gerötet & gerötet \\
\hline & tränend & tränend \\
\hline Datum/Zeit: & $\begin{array}{l}\text { fliessend, weisses } \\
\text { Sekret }\end{array}$ & $\begin{array}{l}\text { fliessend, weisses } \\
\text { Sekret }\end{array}$ \\
\hline \multirow{2}{*}{$\begin{array}{l}\text { Lage } \\
\text { Magensonde } \\
\text { re li }\end{array}$} & $\begin{array}{l}\text { fliessend, gelbes } \\
\text { Sekret }\end{array}$ & $\begin{array}{l}\text { fliessend, gelbes } \\
\text { Sekret }\end{array}$ \\
\hline & Andere & Andere \\
\hline
\end{tabular}

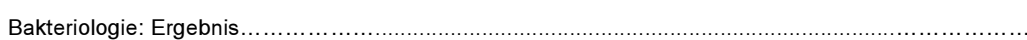

\begin{tabular}{|c|c|c|c|c|}
\hline & Auge Rechts & Auge Links & \\
\hline \multirow{4}{*}{$\begin{array}{c}\text { Beurteilung } \\
\text { (24 St.) }\end{array}$} & gerötet & $\begin{array}{l}\text { abnehmend } \\
\text { zunehmend } \\
\text { gleich }\end{array}$ & gerötet & $\begin{array}{c}\text { abnehmend } \\
\text { zunehmend } \\
\text { gleich }\end{array}$ \\
\cline { 2 - 5 } Datum/Zeit: & tränend & $\begin{array}{l}\text { abnehmend } \\
\text { zunehmend } \\
\text { gleich }\end{array}$ & tränend & $\begin{array}{c}\text { abnehmend } \\
\text { zunehmend } \\
\text { gleich }\end{array}$ \\
\cline { 2 - 5 } & $\begin{array}{c}\text { fliessend, weisses } \\
\text { Sekret }\end{array}$ & $\begin{array}{l}\text { abnehmend } \\
\text { zunehmend } \\
\text { gleich }\end{array}$ & $\begin{array}{c}\text { fliessend, weisses } \\
\text { Sekret }\end{array}$ & $\begin{array}{c}\text { abnehmend } \\
\text { zunehmend } \\
\text { gleich }\end{array}$ \\
\cline { 2 - 5 } $\begin{array}{c}\text { Lage } \\
\text { Magensonde } \\
\text { re ii }\end{array}$ & fliessend, gelbes & $\begin{array}{l}\text { abnehmend } \\
\text { zunehmend } \\
\text { gleich }\end{array}$ & fliessend, gelbes & $\begin{array}{c}\text { abnehmend } \\
\text { zunehmend } \\
\text { gleich }\end{array}$ \\
\cline { 2 - 6 } & Anderet & $\begin{array}{l}\text { abnehmend } \\
\text { zunehmend } \\
\text { gleich }\end{array}$ & Andere & $\begin{array}{c}\text { abnehmend } \\
\text { zunehmend } \\
\text { gleich }\end{array}$ \\
\hline
\end{tabular}

\begin{tabular}{|c|c|c|c|c|}
\hline & Auge Rechts & & Auge Links & \\
\hline \multirow{3}{*}{$\begin{array}{l}\text { Beurteilung } \\
\text { (48 St.) } \\
\text { Datum/Zeit: }\end{array}$} & gerötet & $\begin{array}{l}\text { abnehmend } \\
\text { zunehmend } \\
\text { gleich }\end{array}$ & gerötet & $\begin{array}{l}\text { abnehmend } \\
\text { zunehmend } \\
\text { gleich }\end{array}$ \\
\hline & tränend & $\begin{array}{l}\text { abnehmend } \\
\text { zunehmend } \\
\text { gleich }\end{array}$ & tränend & $\begin{array}{l}\text { abnehmend } \\
\text { zunehmend } \\
\text { gleich }\end{array}$ \\
\hline & $\begin{array}{l}\text { fliessend, weisses } \\
\text { Sekret }\end{array}$ & $\begin{array}{l}\text { abnehmend } \\
\text { zunehmend } \\
\text { gleich }\end{array}$ & $\begin{array}{l}\text { fliessend, weisses } \\
\text { Sekret }\end{array}$ & $\begin{array}{l}\text { abnehmend } \\
\text { zunehmend } \\
\text { gleich }\end{array}$ \\
\hline \multirow{2}{*}{$\begin{array}{l}\text { Lage } \\
\text { Magensonde } \\
\text { re li }\end{array}$} & $\begin{array}{l}\text { fliessend, gelbes } \\
\text { Sekret }\end{array}$ & $\begin{array}{l}\text { abnehmend } \\
\text { zunehmend } \\
\text { gleich }\end{array}$ & $\begin{array}{l}\text { fliessend, gelbes } \\
\text { Sekret }\end{array}$ & $\begin{array}{l}\text { abnehmend } \\
\text { zunehmend } \\
\text { gleich }\end{array}$ \\
\hline & Andere & $\begin{array}{l}\text { abnehmend } \\
\text { zunehmend } \\
\text { gleich }\end{array}$ & Andere & $\begin{array}{l}\text { abnehmend } \\
\text { zunehmend } \\
\text { gleich }\end{array}$ \\
\hline
\end{tabular}

Verbesserung der Symptome nach 48 Stunden

nein

Falls nein: • Bakteriologie abnehmen, Ergebnis:

-Wechsel zu Neosporin-Augentropfen (Ende der Evaluation)

\begin{tabular}{|c|c|c|c|c|}
\hline & Auge Rechts & & Auge Links & \\
\hline \multirow{3}{*}{$\begin{array}{c}\text { Beurteilung } \\
\text { (72 St.) } \\
\text { Datum/Zeit: }\end{array}$} & gerötet & $\begin{array}{l}\text { abnehmend } \\
\text { zunehmend } \\
\text { gleich }\end{array}$ & gerötet & $\begin{array}{l}\text { abnehmend } \\
\text { zunehmend } \\
\text { gleich }\end{array}$ \\
\hline & tränend & $\begin{array}{l}\text { abnehmend } \\
\text { zunehmend } \\
\text { gleich }\end{array}$ & tränend & $\begin{array}{l}\text { abnehmend } \\
\text { zunehmend } \\
\text { gleich }\end{array}$ \\
\hline & $\begin{array}{l}\text { fliessend, weisses } \\
\text { Sekret }\end{array}$ & $\begin{array}{l}\text { abnehmend } \\
\text { zunehmend } \\
\text { gleich }\end{array}$ & $\begin{array}{l}\text { fliessend, weisses } \\
\text { Sekret }\end{array}$ & $\begin{array}{l}\text { abnehmend } \\
\text { zunehmend } \\
\text { gleich }\end{array}$ \\
\hline \multirow{2}{*}{$\begin{array}{l}\text { Lage } \\
\text { Magensonde } \\
\text { re li }\end{array}$} & $\begin{array}{l}\text { fliessend, gelbes } \\
\text { Sekret }\end{array}$ & $\begin{array}{c}\text { abnehmend } \\
\text { zunehmend } \\
\text { gleich }\end{array}$ & $\begin{array}{l}\text { fliessend, gelbes } \\
\text { Sekret }\end{array}$ & $\begin{array}{c}\text { abnehmend } \\
\text { zunehmend } \\
\text { gleich }\end{array}$ \\
\hline & Andere & $\begin{array}{l}\text { abnehmend } \\
\text { zunehmend } \\
\text { gleich }\end{array}$ & Andere & $\begin{array}{l}\text { abnehmend } \\
\text { zunehmend } \\
\text { gleich }\end{array}$ \\
\hline
\end{tabular}

Datum Euphrasiatropfen stopp (Dauer bis zum Abklingen der Symptome):

Bemerkungen:

Bitte ausgefüllte Evaluationsbogen an Lilian Stoffel senden (FK B326) 


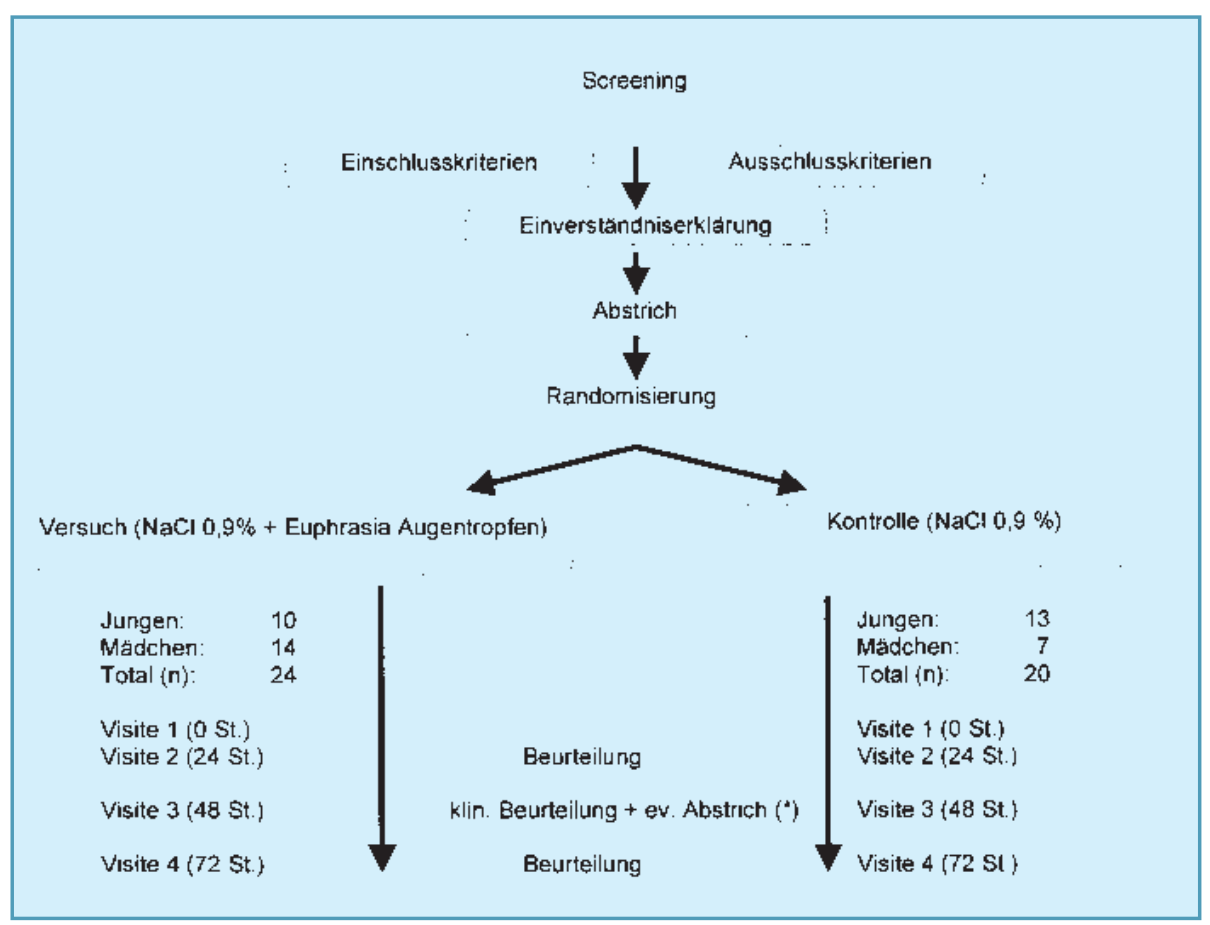

Abb. 2. Flow Chart zum Ablauf des Pilotprojektes

\section{Ergebnisse}

Es wurde eine Untersuchung mit 48 Neugeborenen angestrebt. Die Durchführung erfolgte definitiv bei $44 \mathrm{Neu}$ geborenen, weil die Beobachtungszeit auf ein Jahr limitiert war. In Tabelle 1 werden die deskriptiven Daten zu den Neugeborenen dargestellt. Die Augen wurden bei 24 Neugeborenen mit $\mathrm{NaCl}$ $0,9 \%$ ausgewaschen und anschlies- send wurden Euphrasia-Augentropfen (Versuchsgruppe) verabreicht. Bei den restlichen 20 Neugeborenen wurden die Augen mit $\mathrm{NaCl}$ 0,9\% ausgewaschen (Kontrollgruppe). In 14 Fällen handelte es sich um gesunde Termingeborene (je 7 Neugeborene pro Gruppe). In 26 Fällen handelte es sich um Frühgeborene und in vier Fällen um extrem Frühgeborene $(<1000 \mathrm{~g}$ Geburtsgewicht). In der Gruppe 'extrem
Frühgeborene' erhielten drei Kinder eine systemische Antibiotika-Therapie (zwei in der Versuchsgruppe, ein Kind in der Kontrollgruppe).

Die in der Tabelle 2 dargestellten Ergebnisse zeigen die Abstrich- und Symptomresultate nach 48 Stunden. Nur sieben von 24 Neugeborenen der Versuchsgruppe und drei von 20 Neugeborenen in der Kontrollgruppe wurden aufgrund der Symptomatik und/oder Abstrichresultate mit Neosporin-Augentropfen ${ }^{\circledR}$ behandelt. Bei dem Entscheid, auf eine AntibiotikaBehandlung $\mathrm{zu}$ wechseln, traten folgende Szenarien auf:

\section{Versuchsgruppe}

- 5 Kinder mit klinischer Verschlechterung und positivem Abstrich

- 2 Kinder mit klinischer Verbesserung und positivem Abstrich

\section{Kontrollgruppe}

- 2 Kinder mit klinischer Verschlechterung und negativem Abstrich

- 1 Kind mit klinischer Verbesserung und positivem Abstrich

Bei elf Neugeborenen enthielt der Augenabstrich Staphylococcus aureus. Bei neun dieser Neugeborenen musste jedoch nicht auf eine Antibiotika-haltige Behandlung umgestellt werden, weil die Symptome durch das Auswaschen mit NaCl 0,9\% und/oder Euphrasia-Augentropfen rückläufig waren

Tab. 1. Demographische Daten

\begin{tabular}{|c|c|c|c|}
\hline & $\begin{array}{l}\text { Versuchsgruppe (n=24) } \\
\text { ( } \mathrm{NaCl} 0,9 \%+\text { Euphrasia Augentropfen) }\end{array}$ & $\begin{array}{l}\text { Kontrollgruppe }(\mathbf{n}=20) \\
(\mathrm{NaCl} 0,9 \%)\end{array}$ & Total \\
\hline Mädchen / Knaben (n) & $14 / 10$ & $7 / 13$ & $21 / 23$ \\
\hline $\begin{array}{l}\text { Gestationsalter bei Geburt } \\
\text { (Schwangerschaftswoche = SSW) }\end{array}$ & $\begin{array}{l}25 \text { 3/7 - } 40 \text { 6/7 SSW } \\
\text { (Median: } 34 \text { 5/7) }\end{array}$ & $\begin{array}{l}28 \text { 1/7 - } 40 \text { 6/7 SSW } \\
\text { (Median: } 34 \text { 6/7) }\end{array}$ & $253 / 7-406 / 7 \mathrm{SSW}$ \\
\hline Geburtsgewicht & $\begin{array}{l}625 \mathrm{~g}-4400 \mathrm{~g} \\
\text { (Median: } 2177 \mathrm{~g} \text { ) }\end{array}$ & $\begin{array}{l}985 g-4145 g \\
\text { (Median: } 2700 g \text { ) }\end{array}$ & $625 g-4400 g$ \\
\hline $\begin{array}{l}\text { Aktuelles Gestationsalter } \\
\text { (Schwangerschaftswoche = SSW }\end{array}$ & $\begin{array}{l}271 / 7-410 / 7 \text { SSW } \\
\text { (Median: } 36 \text { 2/7) }\end{array}$ & $\begin{array}{l}301 / 7-41 \text { 0/7 SSW } \\
\text { (Median: } 35 \text { 6/7) }\end{array}$ & $271 / 7-410 / 7 \mathrm{SSW}$ \\
\hline Lebenstag bei Behandlungsbeginn & $\begin{array}{l}2-65 \\
\text { (Median: 8) }\end{array}$ & $\begin{array}{l}2-27 \\
\text { (Median: 5) }\end{array}$ & $2-65$ \\
\hline Frühgeborene >1000 Gramm (n) & 14 & 12 & 26 \\
\hline Extrem Frühgeborene <1000 Gramm (n) & 3 & 1 & 4 \\
\hline Termingeborene (n) & 7 & 7 & 14 \\
\hline Systemische Antibiotika-Therapie (n) & 2 & 1 & 3 \\
\hline
\end{tabular}


Tab. 2. Abstrich- und Symptomresultate nach 48 Stunden

\begin{tabular}{|c|c|c|c|}
\hline Ergebnis & Versuchsgruppe & Kontrollgruppe & Gesamt n \\
\hline Verbesserung der Symptome nach $48 \mathrm{~h}$ & 19 & 16 & 35 \\
\hline Verschlechterung der Symptome nach $48 \mathrm{~h}$ & 5 & 4 & 9 \\
\hline Abstrichresultate ohne Befund & 12 & 12 & 24 \\
\hline Abstrichresultate mit Befund & 12 & 8 & 20 \\
\hline Wechsel auf Neosporin aufgrund nur Symptomatik & 0 & 2 & 2 \\
\hline Wechsel auf Neosporin aufgrund nur Abstrichresultat & 2 & 0 & 2 \\
\hline Wechsel auf Neosporin aufgrund Symptomatik und Abstrichresultat & 5 & 1 & 6 \\
\hline Wechsel auf Neosporin gesamthaft & 7 & 3 & 10 \\
\hline Total Neugeborene pro Gruppe & 24 & 20 & 44 \\
\hline
\end{tabular}

und eine deutliche Besserung eintrat, bevor die Abstrichresultate vorlagen. Die Ergebnisse der Augenabstriche werden in Tabelle 3 dargestellt.

Bei einem Neugeborenen der Versuchsgruppe wurde aufgrund eines positiven Augenabstriches mit Escheria Coli auf Neosporin-Augentropfen ${ }^{\circledR}$ gewechselt. Direkt vor dem Wechsel zu den antibiotischen Augentropfen wurde erneut ein Abstrich abgenommen. In diesem Abstrich wurden keine Bakterien mehr nachgewiesen.

Bei keinem der untersuchten Neugeborenen wurden unerwünschte Nebenwirkungen beobachtet.

\section{Diskussion}

Die Resultate zeigen, dass auch bei positiven bakteriologischen Abstrichen mit $\mathrm{NaCl}$ 0,9\% und/oder EuphrasiaAugentropfen gute Ergebnisse erzielt werden konnten. Vor allem bei Staphylococcus aureus konnte in den meisten Fällen auf eine AntibiotikaTherapie verzichtet werden, da die klinischen Symptome bis zum Eintreffen der Abstrichresultate deutlich abgeklungen waren. Bei einem Neugeborenen wurde aufgrund eines positiven Augenabstriches mit Escheria Coli auf Neosporin-Augentropfen ${ }^{\circledR}$ gewechselt. Direkt vor dem Wechsel zu den antibiotikahaltigen Augentropfen wurde entsprechend des Behandlungsprotokolls erneut ein Abstrich abgenommen. In diesem Abstrich wurden keine Bakterien mehr nachgewiesen. Das

Tab. 3. Abstrich-Ergebnisse

\begin{tabular}{|lccc}
\hline Abstrich-Ergebnisse & $\begin{array}{c}\text { Versuchsgruppe } \\
(\mathbf{n}=24)\end{array}$ & $\begin{array}{c}\text { Kontrollgruppe } \\
(\mathbf{n}=20)\end{array}$ & $\begin{array}{c}\text { Total } \\
(\mathbf{n}=\mathbf{4 4})\end{array}$ \\
\hline Ergebnis negativ & $\mathbf{1 2}$ & $\mathbf{1 2}$ & $\mathbf{2 4}$ \\
\hline Ergebnis positiv & $\mathbf{1 2}$ & $\mathbf{8}$ & $\mathbf{2 0}$ \\
\hline$(+)$ Staphylococcus aureus & 6 & 5 & 11 \\
\hline$(+)$ Escheria coli & 2 & 0 & 2 \\
\hline$(+)$ Hämophilus Influenza & 1 & 1 & 2 \\
\hline$(+)$ Neisseria & 0 & 1 & 1 \\
\hline$(+)$ Staphylococcus lugdunensis & 1 & 0 & 1 \\
\hline$(+)$ Gram positive Kokken & 1 & 1 & 2 \\
\hline$(+)$ Streptococcus B & 1 & 0 & 1 \\
\hline
\end{tabular}

heisst, dass die Therapie mit Euphrasia-Augentropfen oder das regelmässige Auswaschen der Augen bereits die Keime eliminiert hatte.

Eine Limitation dieses Pilotprojektes zeigt sich in den Strukturunterschieden sowohl bei den Neugeborenen als auch bei den Augenabstrichresultaten. In der Versuchsgruppe wiesen die Neugeborenen ein tieferes Geburtsgewicht und ein tieferes Gestationsalter auf und waren tendenziell klinisch instabiler.

Die Abstrichresultate enthielten verschiedene Bakterienarten. Die meisten traten höchstens einmal pro Gruppe auf (6 verschiedene Arten in der Versuchsgruppe und vier verschiedene in Kontrollgruppe). Dies und die
Strukturunterschiede könnten mögliche Gründe sein, dass in der Versuchsgruppe häufiger auf Neosporin-Augentropfen ${ }^{\circledR}$ gewechselt wurde.

Das eingesetzte Ablaufschema beinhaltete in der Versuchs- und Kontrollgruppe einen Wechsel zu NeosporinAugentropfen ${ }^{\circledR}$, wenn innerhalb von 48 Stunden keine Besserung der Augensymptomatik eintrat. Diese 48 Stunden wurden aus ethischen Überlegungen heraus gewählt, obwohl in der Literatur eine längere Zeitdauer bis zur Feststellung einer eindeutigen Besserung dokumentiert ist $[9,10]$. Es stellt sich die Frage, ob der hier gewählte Zeitraum von 48 Stunden für eine optimale Beurteilung der Studienergebnisse nicht zu kurz war. 


\section{Empfehlungen}

Aufgrund der Ergebnisse des vorliegenden Pilotprojektes wird auch bei einem positiven Augenabstrich eine Therapie mit einem Antibiotikum als nicht immer notwendig erachtet. Jedoch muss die klinische Situation des Neugeborenen immer mitbeachtet werden.

Bei geröteten, tränenden oder fliessenden Augen oder Bildung von weissem oder gelbem Sekret empfiehlt das Autorenteam:

- Augen mit NaCl 0,9\% auswaschen.

- Bei Zunahme der Symptome innerhalb (48-)72 Stunden Wechsel zu Euphrasia-Augentropfen mit vorausgehenden Abstrichen (Bakteriologie und Chlamydien), v.a. bei Frühgeborenen <1500 Gramm. Dabei muss die klinische Situation des Patienten mitbeachtet werden.

- In Absprache mit dem ärztlichen Dienst erfolgt der Wechsel zu Neosporin-Augentropfen - je nach Abstrichresultat und klinischem $\mathrm{Zu}$ stand des Neugeborenen - oder die lokale Applikation von EuphrasiaAugentropfen wird fortgesetzt.

Die Autoren empfehlen abschliessend, dieses Pilotprojekt in eine randomisierte kontrollierte prospektive Untersuchung auszubauen. Das vorliegende Erhebungs- und Behandlungsprotokoll hat sich für die Praxis als sinnvoll erwiesen.

\section{Literatur}

1. Frauenklinik: Jahresbericht 2004. Frauenklinik Inselspital Bern, 2004.

2. Nelle, M.: Heranreifende Pflege und Medizin in der Neonatologie oder Klinische Spitzenmedizin im Umgang mit zu früh geborenen Kindern. Ärztemagazin Inselspital, 2005.

3. Kraemer, R.: Schöni, M.H.: Berner Daten Buch Pädiatrie. Verlag Hans Huber, Bern 2005.

4. Morant, J.; Ruppanner, H.: Arzneimittel Kompendium der Schweiz ${ }^{\circledR}$. Verlag Documed, Basel 2007.

5. Sheikh, A.; Hurwitz, B.: Topical antibiotics for acute bacterial conjunctivitis: Cochrane systematic review and meta-analysis update. $\mathrm{Br}$ J Gen Pract. 2005 Dec;55(521):962-4.

6. Sheikh, A.; Hurwitz, B.: Antibiotics versus placebo for acute bacterial conjunctivitis. Cochrane Database Syst Rev. 2006 Apr 19; (2):CD001211.
7. Everitt, H.; Little, P.; Smith, P.: A randomised controlled trial of management strategies for acute infective conjunctivitis in general practice. BMJ 2006; 333:321(12 August), doi: 10.1136/bmj.38891.551088.7C (published 17 July 2006).

(http://bmj.bmijournals.com/cgi/content/full/3 33/7563/321)

8. The Complete German Commission E Monographs: Therapeutic Guide to Herbal Medicines. American Botanical Council Austin, Texas. Published in cooperation with Integrative Medicine Communications Boston, Massachusetts, 1998.

9. Ramos, M.: Euphrasia bei Bindehautentzündungen. WELEDA Ärzteforum, 2005;13:6-7.

10. Stoss, M.; Michels, C.; Peter, E.: Beutke R.; Gorter, R: Prospective Cohort Trial of Euphrasia Single-Dose Eye Drops in Conjunctivitis. The Journal of alternative and Complementary Medicine, 2000;6(6):499-508.

\section{Korrespondenzadresse:}

\section{Lilian Stoffel}

dipl. Pflegefachfrau in Pädiatrie und Pflegeexpertin HöFa2 für Neonatologie Medizinische Universitäts-Kinderklinik Inselspital Bern, Abteilung für Neonatologie Effingerstrasse 102, CH-3010 Bern

liliane.stoffel@insel.ch 